\title{
Síndrome cardio-facio-cutáneo: reporte de caso
}

\author{
Cardio-facio-cutaneous syndrome: a case report
}

\author{
María A. Acosta-Aragón PhD ${ }^{1}$, Nelson F. Sotelo-Muñoz MD², \\ Oscar O. Gutiérrez-Montúfar MD2, Diana M. Chicué-Ossa MD², \\ Manuel S. Ordóñez-Bastidas MD
}

\begin{abstract}
Resumen: el síndrome cardio-facio-cutáneo es una entidad clínica y genéticamente heterogénea, perteneciente a un grupo de síndromes conocidos como RASopatías. Este trastorno es de baja prevalencia, con alrededor de 200 a 300 casos en el mundo, e incluye entre sus manifestaciones clínicas rasgos faciales dismórficos, defectos cardíacos y alteraciones cutáneas. Los hallazgos fenotípicos del síndrome cardio-facio-cutáneo que se comparten con otros síndromes y la ausencia de criterios diagnósticos o signos patognomónicos lo convierten en un reto diagnóstico. En este manuscrito se presenta un caso confirmado de síndrome cardio-facio-cutáneo por estudios de genética molecular en una paciente de siete años de edad, mediante el cual se exponen las principales características de esta condición.
\end{abstract}

Palabras clave: síndrome cardio-facio-cutáneo, genes RAS, proteínas RAS, proteínas protooncogénicas $B-R A F$, RASopatías, informes de casos.

Abstract: The cardio-facio-cutaneous syndrome is a clinically and genetically heterogeneous disorder, belonging to a group of syndromes known as RASopathies. This condition has a low prevalence, with around of 200 to 300 cases in the world, and includes dysmorphic facial features, heart defects, and skin abnormalities among its clinical manifestations. The phenotypic findings of cardio-facio-cutane-

\footnotetext{
${ }^{1}$ Médica y Cirujana, MSc en Biología (área Genética Clínica), PhD en Genética de Poblaciones Humanas y Genética Forense. Profesora titular, Departamento de Pediatría, Facultad de Ciencias de la Salud, Universidad del Cauca. Popayán, Colombia. Correo electrónico: morin1924@gmail.com

2 Médico Interno, Facultad de Ciencias de la Salud, Universidad del Cauca. Popayán, Colombia

${ }^{3}$ Médico, residente de segundo año en Pediatría, Facultad de Ciencias de la Salud, Universidad del Cauca

Conflicto de intereses: los autores declaran que no tienen conflicto de intereses

Medicina \& Laboratorio 2016; 22: 479-486

Módulo 21 (Casos clínicos), número 18. Editora Médica Colombiana S.A. $2016^{\circ}$

Recibido el 11 de septiembre de 2016; aceptado el 23 de octubre de 2016
} 
ous syndrome that are shares with other syndromes and the absence of diagnostic criteria or pathognomonic signs make it a diagnostic challenge. Here its present a confirmed case of cardio-facio-cutaneous syndrome by molecular genetic studies in one seven years old patient, through which are exposed the main characteristics of this condition.

Key words: cardiofaciocutaneous syndrome, RAS genes, RAS proteins, proto-oncogene proteins $B-R A F$, RASopathies, case reports.

\section{Acosta-Aragón MA, Sotelo-Muñoz NF, Gutiérrez-Montúfar OO, Chicué-Ossa}

DM, Ordóñez-Bastidas MS. Síndrome cardio-facio-cutáneo: reporte de caso. Medicina \& Laboratorio 2016; 22: 479-486.

$\mathrm{E}$ síndrome cardio-facio-cutáneo es un trastorno genético, no cromosómico, caracterizado por alteraciones cardíacas, manifestaciones cutáneas, rasgos faciales dismórficos $[1,2]$ y un patrón de herencia autosómico dominante con penetrancia completa [3]. La mayor parte de los casos son causados por mutaciones de novo de sentido erróneo en genes que codifican para proteínas que hacen parte de la vía RAS/MAPK, también alteradas en el síndrome de Noonan y el síndrome de Costello [4,5]; razón por la que se conocen como RASopatías [2]. Las alteraciones producidas en las proteínas de esta vía molecular afectan la señalización de procesos celulares importantes como la proliferación, la diferenciación y la apoptosis [6].

Como genes responsables del síndrome cardio-facio-cutáneo se han descrito: $B R A F(80 \%$ de los casos), MAP2K1, MAP $2 K 2$ y $K R A S[2,3,7]$, lo que lo diferencia de los síndromes de Noonan y Costello, en los que se encuentran implicados los genes PTPN11 y HRAS, respectivamente [6]. Se estima que en el mundo existen entre 200 y 300 personas afectadas con el síndrome cardio-facio-cutáneo [2] y que su prevalencia es de aproximadamente 1 caso por cada 810.000 habitantes en Japón; no obstante, se considera que se encuentra altamente subdiagnosticado, por lo cual su prevalencia a nivel mundial es desconocida [7].

Las alteraciones cutáneas están presentes en el 100\% de las personas afectadas con el síndrome cardio-facio-cutáneo y exhiben una amplia variabilidad clínica, entre las que se incluyen: hiperqueratosis palmoplantar, queratosis pilar, ictiosis, eccema no seborreico, manchas café con leche, nevus pigmentado, piel marmórea y linfedema $[1,3,6]$. A su vez, las principales manifestaciones faciales son: macrocefalia relativa, frente alta, sienes estrechas, ptosis palpebral, hipertelorismo, pliegues epicánticos, puente nasal ancho, surco nasolabial profundo, mentón pequeño y orejas anguladas hacia atrás $[1,3]$. Alrededor del $75 \%$ de los pacientes presentan alguna anomalía cardíaca [6], con mayor frecuencia estenosis de la válvula pulmonar, seguida de miocardiopatía hipertrófica y defectos septales [7]. Además, suele acompañarse de retraso global en el neurodesarrollo, problemas 
oftalmológicos, como nistagmo, estrabismo o atrofia óptica, y alteraciones gastrointestinales como el reflujo gastroesofágico $[1,6,8]$.

En este manuscrito se presenta un caso confirmado de síndrome cardio-facio-cutáneo por estudios de genética molecular en una paciente de siete años de edad, en la que se revelan las principales características de esta condición.

\section{Caso clínico}

Paciente de siete años, sexo femenino, procedente del área urbana de la ciudad de Popayán (Colombia). Hija de una madre de 28 años (en el momento del nacimiento), con embarazos: 2, partos: 2, abortos: 0, producto de la segunda gestación. El embarazo fue de 39 semanas, sin antecedente de exposición a drogas, agentes físicos o químicos, con hallazgos ecográficos prenatales de macrosomía fetal. El parto fue institucional sin complicaciones, el peso al nacer 4.290 gramos y la talla $52 \mathrm{~cm}$.

A la edad de tres meses fue diagnosticada con enfermedad por reflujo gastroesofágico y bajo peso. A los ocho meses se le detectó retraso en el neurodesarrollo. Al año de edad se reconoció la incapacidad para soportar su propio peso o mantenerse de pie y la ausencia de pronunciación de monosílabos; además, se identificaron rasgos faciales dismórficos. A los dos años y medio de edad la paciente logra la bipedestación; a los cuatro años marcha tambaleante y pronuncia disílabos; a los cinco años el lenguaje es comprensible con las primeras palabras. Actualmente (siete años) presenta disartria, comprende órdenes y se comunica predominantemente con lenguaje no verbal.

Antecedentes de alimentación: lactancia materna exclusiva hasta los 12 meses y ablactación a los tres años y medio. La dieta actual se encuentra basada en suplemento nutricional; la paciente no deglute alimentos que tengan consistencia sólida o textura áspera. Otros antecedentes incluyen: insuficiencia leve y estenosis leve de la válvula pulmonar, hipermetropía, estrabismo divergente y nistagmo. En la revisión por sistemas se encontró: crecimiento rápido de uñas y sudoración nocturna profusa acompañada de prurito generalizado. No existen antecedentes familiares de enfermedades o condiciones genéticas.

En el examen físico se observó una paciente de constitución delgada y con baja talla para la edad (véase figura 1 ). Se registró peso: $15 \mathrm{~kg}$, talla: $100 \mathrm{~cm}$, Índice de Masa Corporal (IMC)/edad: 0, -1 desviaciones estándar, talla/edad: <-2 desviaciones estándar [9]. En la exploración de la cabeza se encontró constricción bitemporal, arcos superciliares hipoplásicos, cabello delgado, ondulado en región superior y regiones laterales de la cabeza, rizado en la región occipital, borde de implantación posterior bajo, pabellones auriculares rotados posteriormente y de implantación baja con dismorfismo, hélices prominentes, ptosis palpebral, pliegue epicántico, fisuras palpebrales dirigidas hacia abajo, estrabismo divergente, agu- 
deza visual corregida con anteojos, puente nasal deprimido, paladar ojival, mala oclusión dental y bruxismo; el cuello es corto y ancho (véanse figuras 2 y 3 ).

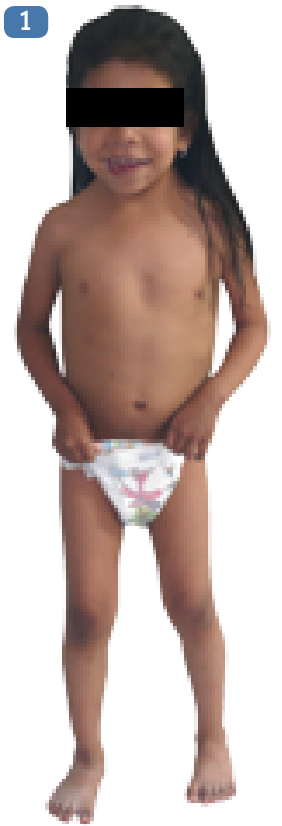

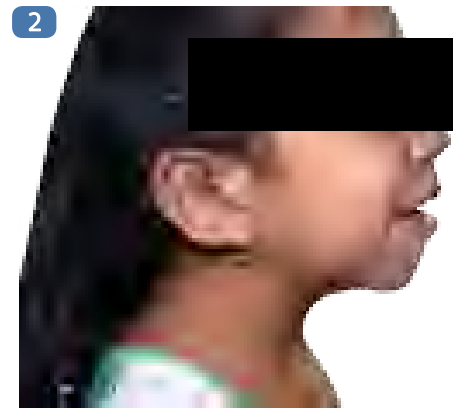

Figura 2. Vista lateral de paciente con síndrome cardio-facio-cutáneo en la cual se observan cambios en las características del cabello, lacio en la mayoría de su zona de crecimiento y rizado en la zona occipital. El área de la piel es hipocrómica en la comisura labial derecha y en los submandibulares. Además, la implantación de las orejas es baja con dismorfismo y rotación posterior.

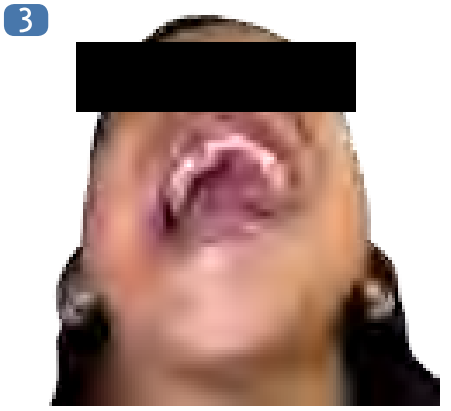

Figura 3. Vista anterior de paciente con síndrome cardio-facio-cutáneo en la cual se observa apertura oral con paladar alto, malposición dental de la pieza 52, cuello corto $y$ ancho.

Figura 1. Paciente con síndrome cardio-facio-cutáneo. Obsérvense facies dismórficas, implantación anterior del cabello baja, relación de segmentos 1,08 , pectus excavatum y pie plano bilateral.

En la valoración de la piel se visualizaba xerosis generalizada, queratosis pilar facial con área hipomelanótica en comisura labial y surco nasogeniano derecho, hemangioma plano en cuello, múltiples nevus melanocíticos en tronco y extremidades, y manchas hiperpigmentadas en extremidades y región vulvar. En la evaluación del sistema osteo-artro-muscular se detectaron pectus excavatum, cifosis dorsal, asimetría en la marcha con pie plano bilateral y pie equino varo izquierdo. A nivel neurológico se observaba hipotonía de extremidades, defensa táctil e hipersensibilidad en cavidad oral y planta de los pies y ausencia del control de los esfínteres. La expresión oral se limitaba a un conjunto reducido de palabras, aunque no existía déficit aparente en la comprensión de las órdenes y las ideas.

Los hallazgos clínicos encontrados motivaron a la valoración por genética clínica y la realización de exámenes complementarios, entre los cuales se obtuvieron un cariotipo 46XX, con el cual se descartó un síndrome de Turner. Se efectuó un análisis de secuenciación para los genes PTPN1 y SOS1, relacionados con el síndrome de Noonan, HRAS, relacionado con el síndrome de Costello, y los genes KRAS, $M A P 2 K 2$ y $R A F 1$, sin detectar mutaciones en alguno de ellos. En el análisis de secuenciación del gen $B R A F$ se identificó una mutación $1802 \mathrm{~A}>\mathrm{T}$ de carácter heterocigoto. Los análisis genéticos en ambos padres no demostraron esta mutación. 
Otros estudios realizados fueron tomografía axial computarizada (TAC) cerebral y radiografías de columna, reja costal, columna total y comparativa de manos, los cuales fueron reportados como normales. Finalmente, las características fenotípicas y clínicas encontradas en la paciente y el resultado de los estudios de genética molecular permitieron establecer el diagnóstico del síndrome cardio-facio-cutáneo.

\section{Discusión}

El síndrome cardio-facio-cutáneo constituye una entidad genéticamente heterogénea que comparte características clínicas con otras RASopatías, particularmente con los síndromes de Noonan y Costello [2]. Por ello, desde su primera descripción en 1986 hubo controversia en relación con su establecimiento como un síndrome nuevo [10]; sin embargo, estudios realizados en la década pasada permitieron demostrar que el síndrome cardio-facio-cutáneo y los síndromes de Noonan y Costello sí eran condiciones distintas aunque con fenotipos superpuestos y que la similitud entre ellos yace en que los genes comprometidos hacen parte de la misma vía molecular [7]. Las características fenotípicas en común con otros síndromes y la amplia variabilidad de las manifestaciones clínicas descritas en el síndrome cardio-facio-cutáneo hace que en algunos casos llegar al diagnóstico pueda representar un verdadero desafío.

Aunque hasta el momento se han realizado pocos estudios de correlación entre el genotipo y el fenotipo en el síndrome cardio-facio-cutáneo, una asociación estadísticamente significativa es la incidencia de estenosis pulmonar, la cual está presente en el $50 \%$ de los individuos con una mutación en el gen BRAF y en el $37 \%$ de aquellos con una mutación en los genes MAP2K1 o MAP2K2 [3,7]. Por otro lado, se ha establecido que las mutaciones encontradas en el síndrome cardio-facio-cutáneo y el síndrome de Costello se relacionan con mayor deterioro en el funcionamiento intelectual y adaptativo que las mutaciones descritas en el síndrome de Noonan [8].

En el caso presentado los principales hallazgos clínicos, que hacen parte del síndrome cardio-facio-cutáneo, fueron los rasgos craneofaciales característicos, las alteraciones cutáneas, la baja estatura, los defectos oculares, las alteraciones musculoesqueléticas, el retraso en el desarrollo del lenguaje verbal y los problemas gastrointestinales, que incluyen las dificultades en la alimentación. En relación con las manifestaciones en la piel, aunque la severidad y el tipo de alteraciones pueden variar, estas son consideradas características cardinales del síndrome cardio-facio-cutáneo [1]. En este contexto también se ha descrito sudoración excesiva y rápido crecimiento de las uñas $[3,7]$, manifestaciones presentes en el caso descrito.

En cuanto a la baja estatura, esta es una característica común en todas las RASopatías y se estima que dos tercios de los niños con síndrome cardio-facio-cutáneo la 
presentan [11]. A pesar de que su causa exacta no está bien establecida, al menos, en parte, se puede relacionar con los problemas en la alimentación, representados por la aversión oral y la disfunción en la deglución [3,7]. De igual modo, los trastornos musculoesqueléticos también están presentes en todas las RASopatías, pero son particularmente prominentes en el síndrome cardio-facio-cutáneo, en donde ocurren con una alta frecuencia y causan discapacidad significativa $[1,7]$.

Respecto a las habilidades para la comunicación se ha observado que el lenguaje receptivo está típicamente más desarrollado que el lenguaje expresivo y se conoce que en promedio los niños con síndrome cardio-facio-cutáneo pronuncian su primera palabra alrededor de los dos años de edad, aunque entre el $9 \%$ y el $31 \%$ permanecen sin comunicación verbal [1]. En conjunto, las alteraciones neurológicas están presentes de forma universal en el síndrome cardio-facio-cutáneo y varían de leves a severas $[1,3,7]$. Conviene mencionar a la hipotonía por su relación con el retraso motor, el cual se hace evidente, por ejemplo, a una edad promedio para caminar sin asistencia de aproximadamente tres años y teniendo en cuenta que hasta el $18 \%$ de los individuos con este síndrome son incapaces de lograr una marcha independiente $[1,7,12]$.

Es importante destacar que actualmente no existen criterios diagnósticos establecidos para el síndrome cardio-facio-cutáneo y no se han descrito manifestaciones patognomónicas o características obligatorias que puedan ayudar a hacer un diagnóstico clínico definitivo [2,3]. Además de las manifestaciones clínicas expuestas, los defectos cardíacos son las alteraciones más representativas entre los hallazgos identificados mediante exámenes complementarios [1]. La estenosis valvular pulmonar es el más común $[1-3,7]$ y fue documentada en la paciente de este caso a través de ecocardiografía. Por su parte, las arritmias no son usuales en el síndrome cardio-facio-cutáneo, a diferencia del síndrome de Costello en donde ocurren con mayor frecuencia [13].

En discrepancia con las características clínicas del síndrome cardio-facio-cutáneo la paciente de este caso no presentaba convulsiones, las cuales, en contraste con otras RASopatías, son consideradas una manifestación común de este síndrome, caracterizadas por ser a menudo refractarias al tratamiento, lo que hace necesario el uso concomitante de diferentes anticonvulsivantes $[3,7,14]$.

De acuerdo a lo anterior, como consecuencia de la dificultad para realizar un diagnóstico correcto del síndrome cardio-facio-cutáneo basado únicamente en las características fenotípicas, es necesario realizar la confirmación por estudios de genética molecular que permitan identificar mutaciones en alguno de los cuatro genes actualmente reconocidos como asociados a este síndrome [1-3,7]. En la paciente del presente caso estas pruebas reportaron una mutación de sentido erróneo en el gen $B R A F$; una secuencia conformada por aproximadamente 190 $\mathrm{kb}$ [3]. Las mutaciones en este gen son las más comunes en el síndrome cardio- 
facio-cutáneo y no están asociadas con los síndromes de Noonan y Costello [3,7]. Además, se acepta que los individuos con variantes patogénicas del gen BRAF tienen un diagnóstico de síndrome cardio-facio-cutáneo, incluso si presentan características descritas para el síndrome de Costello o un fenotipo sobrepuesto con el síndrome de Noonan $[3,4]$.

En otro sentido, y como parte del diagnóstico diferencial, es necesario tener en cuenta que en el síndrome de Noonan existe una menor incidencia del retraso cognitivo severo y de las alteraciones cutáneas, mientras que en el síndrome de Costello se presentan algunas características particulares que no se observan en el síndrome cardio-facio-cutáneo como son los papilomas en el rostro o la región perianal, la piel flácida y la desviación ulnar de la muñeca y los dedos $[3,7]$. Finalmente, los tumores malignos observados en otras RASopatías, incluyendo los síndromes de Noonan y Costello, no parecen ser una característica principal del síndrome cardio-facio-cutáneo [2].

\section{Conclusiones}

Dada la variabilidad del síndrome cardio-facio-cutáneo en su expresión clínica existe una alta posibilidad de que sea interpretado como otra entidad; por lo tanto, es evidente la necesidad de realizar estudios de genética molecular que permitan identificar una mutación en alguno de los cuatro genes actualmente implicados y así confirmar el diagnóstico sospechado. La descripción de este síndrome tan poco común e infrecuente hace que su presentación sea una valiosa oportunidad para conocer las principales características que han sido descritas en la literatura. El diagnóstico temprano de esta condición permite el inicio oportuno de intervenciones multidisciplinares que impactan en el curso de la enfermedad, particularmente en la calidad de vida de los pacientes y su familia, a la vez que se previenen complicaciones y se promueve el desarrollo motor e intelectual del paciente.

\section{Agradecimientos}

A la paciente y su familia por su plena colaboración y autorización para compartir su caso en la literatura científica.

\section{Bibliografía}

1. CFC international. Understanding Cardio-Facio-Cutaneous Syndrome: A Guide for Parents, Providers, and Educators. Nueva York, Estados Unidos. 2015. Disponible: https://rasopathiesnet. org/wp-content/uploads/2014/01/CFC-ParentGuide_R4.pdf. Consultado: oct 2016.

2. U.S. Department of Health \& Human Services, National Institutes of Health, National
Library of Medicine, Lister Hill National Center for Biomedical Communications. Cardiofaciocutaneous syndrome. Maryland, Estados Unidos. 2012. Disponible: https://ghr.nlm.nih.gov/ condition/cardiofaciocutaneous-syndrome. Consultado: oct 2016.

3. Rauen KA. Cardiofaciocutaneous Syndrome. En: Pagon RA, Adam MP, Ardinger HH, Wallace SE, 
Amemiya A, Bean LJ, et al., eds. GeneReviews $\AA$. Washington, Estados Unidos: University of Washington; 2016.

4. Kavamura MI, Neri G. Cardiofaciocutaneous syndrome. Orphanet. 2015. Disponible: http://www.orpha.net/consor/cgi-bin/OC_Exp. php?Lng=EN\&Expert $=1340 \#$. Consultado: oct 2016.

5. Ciara E, Pelc M, Jurkiewicz D, Kugaudo $M$, Gieruszczak-Bialek D, Skorka A, et al. Is diagnosing cardio-facio-cutaneous (CFC) syndrome still a challenge? Delineation of the phenotype in 15 Polish patients with proven mutations, including novel mutations in the BRAF1 gene. Eur J Med Genet 2015; 58: 14-20.

6. Roberts A, Allanson J, Jadico SK, Kavamura MI, Noonan J, Opitz JM, et al. The cardiofaciocutaneous syndrome. J Med Genet 2006; 43: 833-842.

7. Pierpont ME, Magoulas PL, Adi S, Kavamura MI, Neri G, Noonan J, et al. Cardio-facio-cutaneous syndrome: clinical features, diagnosis, and management guidelines. Pediatrics 2014; 134: e1149-1162.

8. Cesarini L, Alfieri P, Pantaleoni F, Vasta I, Cerutti M, Petrangeli V, et al. Cognitive profile of disorders associated with dysregulation of the RAS/MAPK signaling cascade. Am J Med Genet A
2009; 149A: 140-146.

9. República de Colombia, Ministerio de Salud y Protección Social. Gráficas de patrones de crecimiento en niños, niñas y adolescentes. 2015. Disponible: https://www.minsalud.gov.co/salud/ Paginas/primera-infancia-patrones-crecimientoninos-adolescentes.aspx. Consultado: oct 2016.

10. Neri G, Zollino M, Reynolds JF. The NoonanCFC controversy. Am J Med Genet 1991; 39: 367370.

11. Stevenson DA, Yang FC. The musculoskeletal phenotype of the RASopathies. Am J Med Genet C Semin Med Genet 2011; 157C: 90-103.

12. Yoon G, Rosenberg J, Blaser S, Rauen KA. Neurological complications of cardio-facio-cutaneous syndrome. Dev Med Child Neurol 2007; 49: 894-899.

13. Lin AE, Alexander ME, Colan SD, Kerr B, Rauen KA, Noonan J, et al. Clinical, pathological, and molecular analyses of cardiovascular abnormalities in Costello syndrome: a Ras/MAPK pathway syndrome. Am J Med Genet A 2011; 155A: 486-507.

14. Adachi M, Abe Y, Aoki Y, Matsubara Y. Epilepsy in RAS/MAPK syndrome: two cases of cardio-facio-cutaneous syndrome with epileptic encephalopathy and a literature review. Seizure 2012; 21: 55-60.

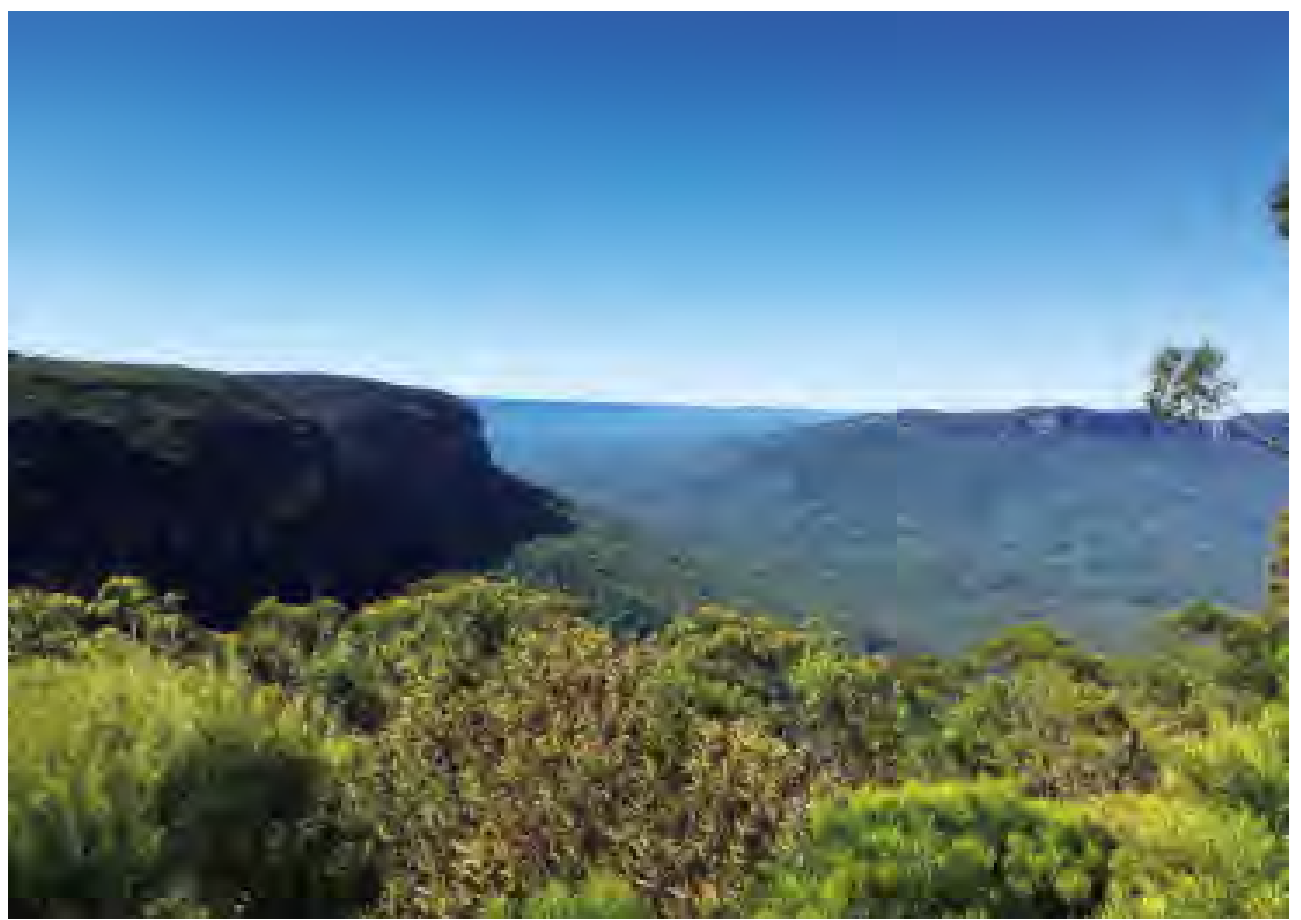

Blue Mountains \& Wildlife Park.

Australia.

Carlos Alberto Lozano M. carlos.lozano@iatm.com.co 